\title{
Three-dimensional evaluation of the transfer accuracy of a bracket jig fabricated using computer-aided design and manufacturing to the anterior dentition: An in vitro study
}

\author{
Jae-Hyun Park ${ }^{\mathrm{a}}$ (1) \\ Jin-Young Choi ${ }^{\mathrm{a}}$ \\ Seong-Hun $\mathrm{Kim}^{\mathrm{a}}$ (1) \\ Su-Jung Kim ${ }^{\mathrm{a}}$ \\ Kee-Joon Lee ${ }^{b}$ \\ Gerald Nelson $^{\mathrm{a}}$
}

${ }^{2}$ Department of Orthodontics, Graduate School of Dentistry, Kyung Hee

University, Seoul, Korea

${ }^{\mathrm{b}}$ Department of Orthodontics, Institute of Craniofacial Deformity, Yonsei University College of Dentistry, Seoul, Korea
Objective: To evaluate the accuracy of a one-piece bracket jig system fabricated using computer-aided design and manufacturing (CAD/CAM) by employing three-dimensional (3D) digital superimposition. Methods: This in vitro study included 226 anterior teeth selected from 20 patients undergoing orthodontic treatment. Bracket position errors from each of the 40 arches were analyzed quantitatively via 3D digital superimposition (best-fit algorithm) of the virtual bracket and actual bracket after indirect bonding, after accounting for possible variables that may affect accuracy, such as crowding and presence of the resin base. Results: The device could transfer the bracket accurately to the desired position of the patient's dentition within a clinically acceptable range of \pm 0.05 $\mathrm{mm}$ and $2.0^{\circ}$ for linear and angular measurements, respectively. The average linear measurements ranged from 0.029 to $0.101 \mathrm{~mm}$. Among the angular measurements, rotation values showed the least deviation and ranged from $0.396^{\circ}$ to $0.623^{\circ}$. Directional bias was pronounced in the vertical direction, and many brackets were bonded toward the occlusal surface. However, no statistical difference was found for the three angular measurement values (torque, angulation, and rotation) in any of the groups classified according to crowding. When the teeth were moderately crowded, the mesio-distal, bucco-lingual, and rotation measurement values were affected by the presence of the resin base. Conclusions: The characteristics of the CAD/CAM one-piece jig system were demonstrated according to the influencing factors, and the transfer accuracy was verified to be within a clinically acceptable level for the indirect bracket bonding of anterior teeth.

[Korean J Orthod 2021;51(6):375-386]

Key words: 3D scanner, Bracket, Bonding, Digital model

Received December 24, 2020; Revised June 5, 2021; Accepted June 7, 2021.

Corresponding author: Seong-Hun Kim.

Professor, Department of Orthodontics, Graduate School of Dentistry, Kyung Hee University, 26 Kyungheedae-ro, Dongdaemun-gu, Seoul 02447, Korea.

Tel +82-2-958-9392 e-mail bravortho@gmail.com

How to cite this article: Park JH, Choi JY, Kim SH, Kim SJ, Lee KJ, Nelson G. Threedimensional evaluation of the transfer accuracy of a bracket jig fabricated using computer-aided design and manufacturing to the anterior dentition: An in vitro study. Korean J Orthod 2021;51:375-386.

(C) 2021 The Korean Association of Orthodontists.

This is an Open Access article distributed under the terms of the Creative Commons Attribution Non-Commercial License (http://creativecommons.org/licenses/by-nc/4.0) which permits unrestricted non-commercial use, distribution, and reproduction in any medium, provided the original work is properly cited. 


\section{INTRODUCTION}

Orthodontists typically endeavor to complete orthodontic treatment with an ideal occlusion that satisfies Andrews' six keys to normal occlusion. ${ }^{1,2}$ In order to achieve this objective, clinicians must place the bracket in the exact location that will position the tooth ideally; however, this is quite challenging and is a frequently discussed topic in the literature. Some clinicians have adopted an indirect bonding system (IDBS) to achieve more precise bracket positioning. ${ }^{3-6}$ Irrespective of how accurately the orthodontist positions a bracket, if an error occurs during its transfer to the tooth, the IDBS cannot produce a better treatment effect than does the direct bonding method. Therefore, the accuracy of the transfer device used to deliver the bracket is of ultimate importance. $^{7,8}$

Many bracket transfer trays or jigs have been developed with various materials and using various methods, and their accuracy has been studied and verified using digital technology. ${ }^{9-12}$ These devices can be fabricated manually in the laboratory, or the entire process can be digitalized using computer-aided design and manufacturing (CAD/CAM) ${ }^{13-16}$ To our knowledge, no previous studies have verified the accuracy of a transfer device fabricated using CAD/CAM, taking into account various possible variables that may affect its accuracy.

Recently, a new type of one-piece CAD/CAM bracket transfer jig system (one-piece jig) has been developed to improve transfer accuracy (Figure 1). ${ }^{12,17}$ It is an improved version of a previously studied two-piece jig design incorporating a process of resin base formation. ${ }^{18}$ The two-piece jig has a part that holds the bracket and teeth separately, and the two parts are connected to perform bracket bonding. ${ }^{18,19}$ This two-piece design results in many transfer errors because the bracket position is guided by inserting a small sectional wire into the bracket slot. The one-piece jig system presented in this article has contours that adapt to all the labial structures of the bracket, as well as to the occlusal surfaces or incisal edges of the teeth. In the clinical setting, some pos- sible variables could affect transfer accuracy: the presence or absence of a resin base, the degree of crowding, and the shape of the cusp. Clinicians are aware that indirect bonding may not result in a consistently thin layer of bonding agent. Irregularities in enamel shape and surface also affect the bracket position. Even when efforts are taken to minimize this gap in the virtual setup process, extra resin may be required to fill tiny gaps. This resin base is a key to successful customization using the conventional IDBS, and it contributes to the stability of jig delivery. ${ }^{18,19}$ Moreover, the CAD/CAMcustomized device used in this study could be affected by the arrangement of the occlusal surfaces or incisal edges. In particular, if the anterior teeth are severely crowded, it may not be possible to accurately fabricate a customized jig owing to misinformation regarding the incisal edges. The cusp height might also affect the accuracy of indirect bracket positioning; however, studies on the posterior teeth and the effect of cusp height on the IDBS have shown that this is not significant. ${ }^{20}$

The primary aim of this in vitro study was to evaluate the bracket transfer accuracy of a newly developed onepiece CAD/CAM transfer jig system to the upper and lower anterior teeth by using three-dimensional (3D) digital superimposition. The secondary goal of this study was to demonstrate the effect of independent clinical variables on transfer accuracy.

\section{MATERIALS AND METHODS}

This study was approved by the Institutional Review Board at the Kyung Hee University Dental Hospital (KHDT19025). For this in vitro study, 6 anterior teeth (from the canine to canine) of 40 dental arches (20 upper and 20 lower arches) were selected from 20 patients undergoing orthodontic treatment (13 women and 7 men; age range: $13-48$ years; average age: 22.3 years). For calculating the sample size (G*Power version 3.1.9.3; Heinrich-Heine-Universität Düsseldorf, Düsseldorf, Germany), a Wilcoxon-Mann-Whitney test (two-tailed) was conducted for an expected $r=0.6$ corresponding to a

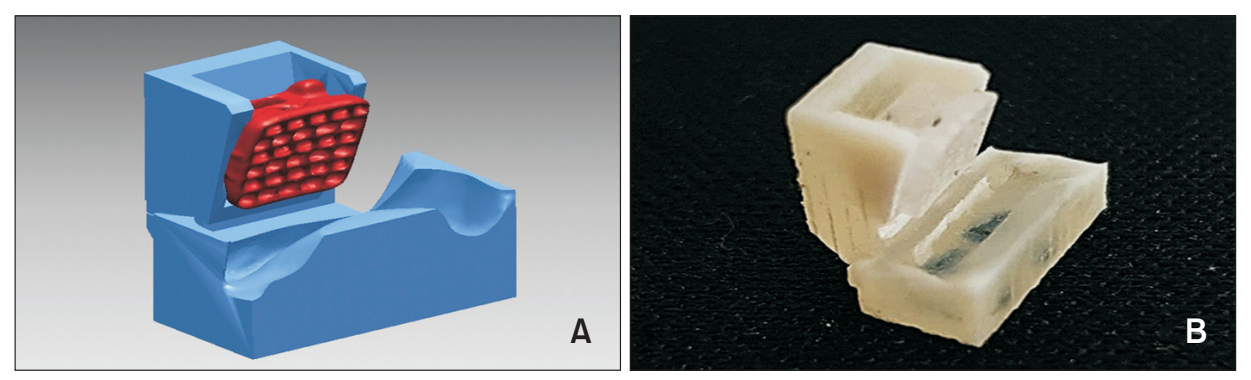

Figure 1. One-piece transfer jig system fabricated using computer-aided design and manufacturing for indirect bonding. A, Virtual one-piece jig with bracket. B, Ceramic bracket adapted to the three-dimensional-printed one-piece jig. 
large size effect. The results revealed a required number of 154 teeth having a type 1 error of 0.05 and 100\% power. The inclusion criteria were as follows: (1) patients scheduled to be treated using the IDBS, (2) with permanent dentition, and (3) intact natural teeth without defects or restorations. Each of the 40 arches was divided into three types based on crowding (mild [type 1], moderate [type 2], and severe crowding [type 3]) according to Little's irregularity index. ${ }^{21}$ This was calculated by adding five measurements of linear displacement of the anatomic contact points of the anterior teeth. According to the index value, a crowding of $\leq 3 \mathrm{~mm}$ was classified as mild (including 4 spaced arches; total 13 arches), 4-8 $\mathrm{mm}$ as moderate (14 arches), and $>8 \mathrm{~mm}$ as severe (13 arches). Brackets that were impossible to be initially bonded to the appropriate positions because of malpositioned jigs in cases of severe crowding were excluded. Finally, 116 and 109 brackets for the upper and lower dentition, respectively, were included.

For all patients, the experiment was performed using brackets with and without the resin base, and the patients were classified into two groups: group A included patients receiving brackets with a resin base and group $\mathrm{B}$ included those receiving brackets without a resin base. The schematic of the study procedure is shown in Figure 2. The initial plaster models of the patients' dentitions were laser scanned (Medit T500; Medit, Seoul, Korea) and converted to stereolithographic files. Using this digital information and the software program (3Txer; Cenos Co., Anyang, Korea), virtual setups were performed and the brackets were positioned precisely on each individual tooth surface (Figure 3A and 3D). Each bracket slot was placed on the plane at the center of the central incisors and canines. The template wire for bracket positioning was established on the 3Txer software, and the locations of the brackets were adjusted to enable the previ- ously customized template wire to pass through. The gap between the bracket base and tooth surface was minimized. Thereafter, the customized one-piece bracket transfer jigs were designed and fabricated using $\mathrm{CAD} /$ CAM and a polyjet-type 3D printer (ProJet MJP 3600; 3D Systems Co., Rock Hill, SC, USA) (Figure 3B and 3E).

The resin bases were additionally fabricated for the brackets in group A by using the following method. Separating agents were applied to the tooth surface of the rapid prototyping $(\mathrm{RP})$ model, and the bracket base was washed and sand-blasted. After assembling the bracket and the jig, the bonding agent (Transbond ${ }^{\mathrm{TM}}$ XT Primer; $3 \mathrm{M}$ Unitek, Monrovia, CA, USA) and resin adhesive

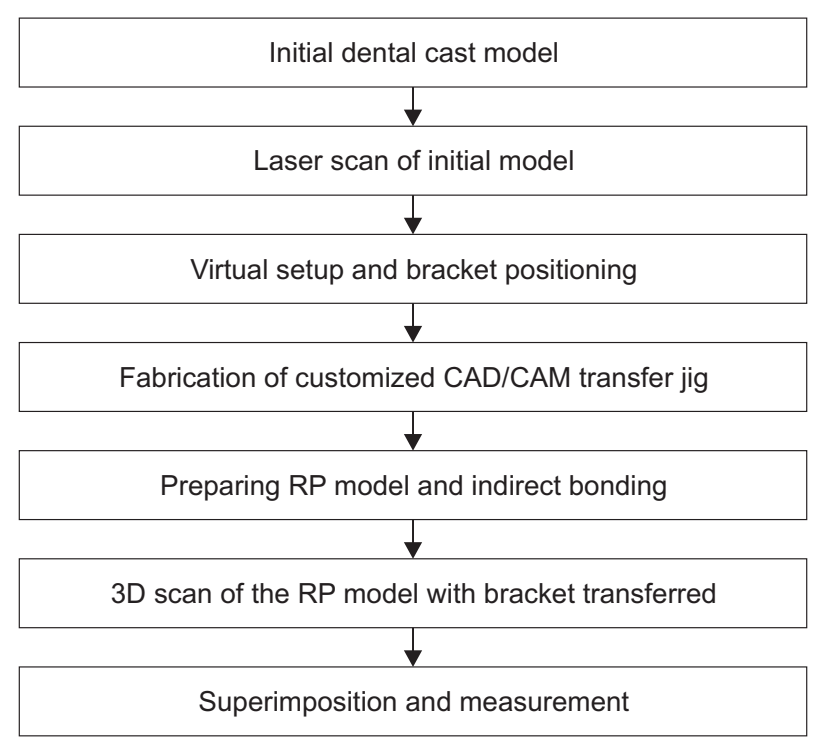

Figure 2. Schematic illustrating the research design. CAD/CAM, computer-aided design and manufacturing; $\mathrm{RP}$, rapid prototyping; 3D, three-dimensional.
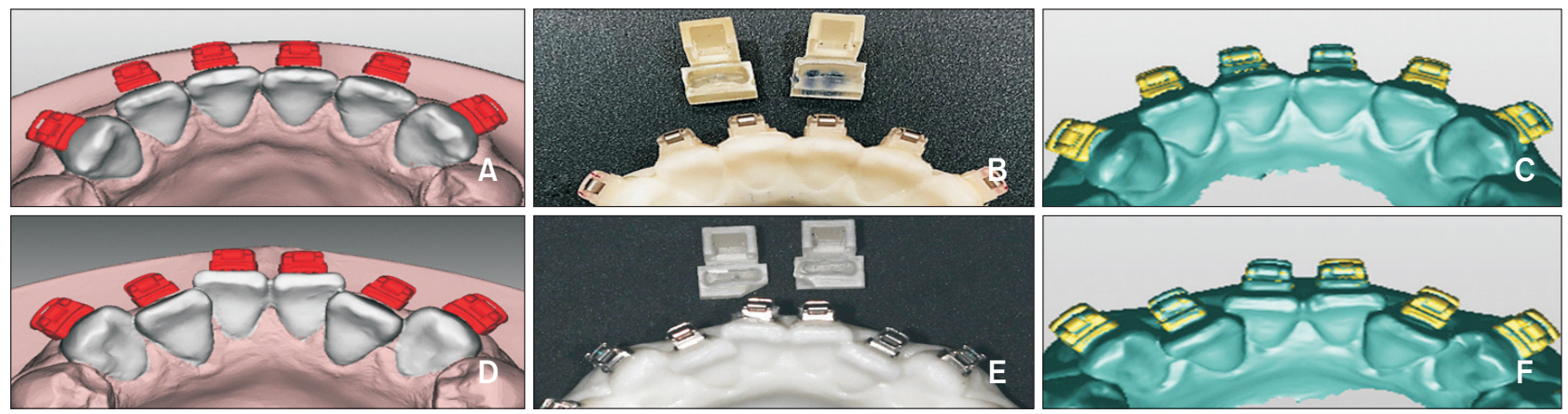

Figure 3. Progress of digital indirect bonding and transfer accuracy evaluation in cases of mild crowding (A-C) and moderate crowding (D-F). A, D, Virtual brackets are positioned precisely on each individual tooth surface through virtual setups with the software program (3Txer; Cenos Co., Anyang, Korea). B, E, The customized one-piece bracket transfer jigs are designed and fabricated using computer-aided design and manufacturing and bonded to the rapid prototyping model. C, F, Three-dimensional digital superimposition of the virtual model. 
(Transbond $^{\mathrm{TM}}$ XT Light Cure Adhesive; 3M Unitek) were applied sequentially to the bracket base. The assembly was placed on the initial RP model, and remnant resin was removed. The remaining resin was then light-cured. For this in vitro experiment, the RP models were prepared using scan data of the previously obtained initial model. One orthodontist (J.H.P.) bonded the brackets with and without the resin base to this RP model using a CAD/CAM transfer jig (Figure 3). The same bonding agent and adhesive resin were used to attach the bracket using a light-curing device (VALO; Ultradent, South Jordan, UT, USA). When placed in the planned position, the jig was pressed gently on the incisal edges during lightcuring. Because of the stiff material of the jig, minimal finger pressure was necessary. The RP model with the transferred bracket was then 3D digitally scanned using a high-resolution intraoral scanner (Trios3; 3Shape, Copenhagen, Denmark). Through this process, 3D information for each bracket position on the RP model was obtained.

In this study, the brackets used were self-ligating brackets with a 0.022 -inch slot Tweemac prescription. ${ }^{22}$ The Tweemac prescription includes a combination of the Roth (maxillary incisors and posterior teeth of both the maxilla and mandible), MBT (mandibular incisors and canine), and Smile (maxillary canine, premolars and mandibular premolars) prescriptions. Forty sets of canine-to-canine ceramic self-ligation brackets (Quicklear $^{\circledR}$; Forestadent, Pforzheim, Germany) were used on the upper and lower dentitions.

Bracket position errors were analyzed quantitatively using 3D digital superimposition (best-fit algorithm) of the virtual bracket and the actual bracket after indirect bonding. The RapidForm software 2006 (INUS Technology, Seoul, Korea) was used to perform the superimposition and measurements (Figure 4A). Three linear differences (mesio-distal [M-D], bucco-lingual [B-L], and occluso-gingival [0-G]) and three angular differences (torque $[T]$, angulation $[A]$, and rotation $[R]$ ) of the bracket position between the virtual and actual models were used to reflect bracket position errors. In order to derive these six measurements, a 3D linear coordinate system ( $x-, y-$, and z-axes) for each tooth based on the midpoint of the bracket base was constructed (Figure 4B). The origin of the coordinate system was set to coincide with the center point of the bracket base. The M-D axis ( $\mathrm{x}$-axis) was determined to be parallel to the bracket slot, the B-L direction (y-axis) was formed by drawing a normal line based on the lingual surface of the bracket slot, and the z-axis ( $0-\mathrm{G}$ direction) was determined to be perpendicular to the plane of the other two axes. The virtual bracket position was considered as the baseline or "zero," and all six discrepancies were measured from the baseline position. The signs (positive or negative) of the measurement values indicated the direction in which the bracket was incorrectly bonded when compared to the reference position of each coordinate axis. A positive value indicated a mesial, buccal, and occlusal displacement. A lingual crown torque, distal tip, or distal-in rotation was also reported as a positive value.

A clinically acceptable range for the measurement values was assumed if it was within a linear displacement of $0.5 \mathrm{~mm}$ and an angular discrepancy of $2^{\circ}$, according to the American Board of Orthodontics Objective Grading System (ABO OGS). ${ }^{23}$ The frequency of brackets placed within this range for each group was calculated based on the ABO OGS criterion. The reference position of each coordinate axis was used to determine the direction. The frequency of this directional bias was also included in the calculation.

\section{Statistical analysis}

All statistical analyses were performed using IBM SPSS Statistics for Windows/Macintosh, Version 22.0 (IBM Corp., Armonk, NY, USA), and were conducted separately for the maxillary and mandibular arches. One researcher (J.H.P.) performed all the measurements and statistical analyses. The normality test of the collected outcomes
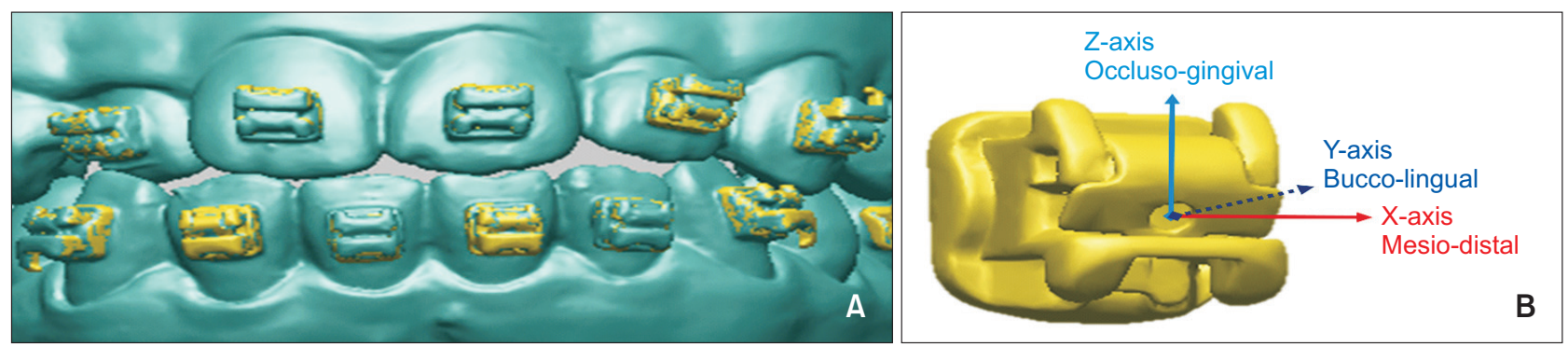

Figure 4. A, Three-dimensional (3D) digital superimposition (best-fit method) of the virtual model (yellow color) and post-transfer model (green color) by using RapidForm software 2006 (INUS Technology, Seoul, Korea). B, The 3D coordinate system of the superimposed bracket. The origin of the coordinate system is set to coincide with the center point of the bracket base. 
was confirmed to follow normal distribution by using the Kolmogorov-Smirnov test $(p>0.05)$. The 3D position of the bracket placed on the virtual model was regarded as the reference. After attaching the bracket to the RP using the custom transfer jig, transfer accuracy was verified by determining how far the bracket was from the reference position. Therefore, a one-sample $t$ test was performed in which the test value was set to zero in each group. An independent $t$-test was used to compare the two independent groups with different resin bases. A one-way analysis of variance (ANOVA) followed by Bonferroni's post hoc test was used for intergroup comparison of the three crowding types. Finally, to evaluate the reproducibility of the six measurement values (M-D, B-L, O-G, T, A, and R), 30 teeth were randomly chosen, and the measurements for each bracket were repeated after an interval of 2 weeks.

\section{RESULTS}

The reproducibility test showed that all six measurements (M-D, B-L, O-G, T, A, and R) were within the one sigma $(1 \sigma)$ range. All of the measurements and statistical results assumed there were no differences between the digital data obtained via laser scanning of the patient's initial cast model and the intraoral scan data of the RP model. ${ }^{24-26}$ For the in vitro study, one of the most important considerations in the interpretation of the entire experimental data was the consistency between the laser scan data and the intraoral scan data. If these two scanned data were superimposed, no difference should be observed. As an indirect method for demonstrating this assumption, the deviation of the two model's digital data at the same location was verified using a onesample $t$-test. Simultaneously, the statistical significance was confirmed on the basis of the verification value of $0.15 \mathrm{~mm}$ with a $10 \%$ zone of equivalence, which is a widely used method in similar studies. Thereafter, we attempted to show that no difference existed between the two datasets by performing an equivalence test (two one-sided $t$-tests). We were able to demonstrate that the data from the two scans were equivalent at a 95\% confidence interval based on the test value with a $10 \%$ margin. Therefore, all the following results were derived assuming that the two datasets were equivalent to each other.

The descriptive statistics and results of the one-sample $t$-test are summarized for each group in Table 1. Notably, a statistically significant difference was observed in the linear measurement value of $0-G$ for each of the experimental groups. In addition, most of the linear and angular measurements showed significant differences, except for rotation. However, this observation did not confirm consistent tendencies between the groups. 
The comparison according to the degree of crowding was performed using one-way ANOVA. The results are summarized in Table 2 for linear and angular differences, respectively, according to Little's irregularity index. Bonferroni's post hoc test results are shown in Table 3 for each experimental group. No significant differences were observed in the angular measurements according to Little's irregularity index in both the groups. For the linear measurements, in group A with a customized resin base, the M-D and $0-G$ differences were statistically significant, as were the differences between types 1 and 2 and between types 1 and 3. In group B without a customized resin base, the B-L and $0-G$ differences were significant.

After controlling for other possible variables, an independent $t$-test was performed on the average of the two groups by using only the presence of the resin base as an independent variable (Table 4). This was done by converting it to an absolute value of measurement. As shown in Table 4, the results differed according to the degree of crowding. In the moderate crowding group, statistically significant differences were found in the values for $\mathrm{M}-\mathrm{D}, \mathrm{B}-\mathrm{L}$, and $\mathrm{R}$ according to the presence or absence of a resin base. In contrast, no differences were observed in all six measurement values in the severe crowding group.

A one-tailed equivalence test, based on a linear measurement of $0.5 \mathrm{~mm}$ and an angular measurement of $2^{\circ}$, was used to determine whether this difference met the ABO OGS criterion. This was done by converting it to an absolute value of measurement. The null hypothesis was that the measured absolute value will be greater than or equal to the value determined using this criterion. Accordingly, the frequency for each of the six dependent variables was expressed as a histogram (Figures 5 and 6). This analysis revealed that all six linear and angular measurements did not significantly exceed the threshold $\left(0.5 \mathrm{~mm}\right.$ and $\left.2^{\circ}\right)$ for each experimental group $(p<$ $0.001)$.

We next examined whether the brackets were bonded with directional bias along each axis of the coordinate system. The results are detailed in Figure 7 . These revealed the existence of a directional bias, except for rotation among the angular measurements. In particular, the bracket bonded more towards the occlusal surface than towards the gingival surface. A large difference in mesiodistal position and torque measurements was also observed. A larger number of brackets was attached in a mesial direction and transferred in a direction that caused the inclination of the crown in a buccal direction.
Table 2. Linear and angular differences according to Little's irregularity index for each experimental group

\begin{tabular}{|c|c|c|}
\hline Variable & Group A & Group B \\
\hline \multicolumn{3}{|c|}{ Linear measurement } \\
\hline \multicolumn{3}{|c|}{ Mesio-distal (mm) } \\
\hline Type 1 & $-0.022 \pm 0.068$ & $0.012 \pm 0.020$ \\
\hline Type 2 & $0.016 \pm 0.016$ & $0.018 \pm 0.044$ \\
\hline Type 3 & $0.005 \pm 0.028$ & $0.006 \pm 0.031$ \\
\hline$p$-value & $<0.001^{* * *}$ & 0.090 \\
\hline \multicolumn{3}{|c|}{ Bucco-lingual (mm) } \\
\hline Type 1 & $0.006 \pm 0.060$ & $0.018 \pm 0.049$ \\
\hline Type 2 & $0.004 \pm 0.050$ & $-0.018 \pm 0.069$ \\
\hline Type 3 & $-0.006 \pm 0.048$ & $0.020 \pm 0.044$ \\
\hline$p$-value & 0.316 & $<0.001^{* * *}$ \\
\hline \multicolumn{3}{|c|}{ Occluso-gingival (mm) } \\
\hline Type 1 & $-0.117 \pm 0.116$ & $-0.125 \pm 0.070$ \\
\hline Type 2 & $-0.064 \pm 0.073$ & $-0.082 \pm 0.089$ \\
\hline Type 3 & $-0.074 \pm 0.090$ & $-0.081 \pm 0.052$ \\
\hline$p$-value & $0.002^{* *}$ & $0.001^{* *}$ \\
\hline \multicolumn{3}{|c|}{ Angular measurement } \\
\hline \multicolumn{3}{|c|}{ Torque $\left(^{\circ}\right)$} \\
\hline Type 1 & $0.167 \pm 0.705$ & $0.143 \pm 0.585$ \\
\hline Type 2 & $0.144 \pm 0.643$ & $-0.018 \pm 0.704$ \\
\hline Type 3 & $0.170 \pm 0.658$ & $0.166 \pm 0.670$ \\
\hline$p$-value & 0.961 & 0.187 \\
\hline \multicolumn{3}{|l|}{ Angulation $\left(^{\circ}\right)$} \\
\hline Type 1 & $-0.164 \pm 0.768$ & $0.034 \pm 0.780$ \\
\hline Type 2 & $-0.095 \pm 0.611$ & $0.003 \pm 0.783$ \\
\hline Type 3 & $-0.105 \pm 0.628$ & $-0.165 \pm 0.728$ \\
\hline$p$-value & 0.732 & 0.254 \\
\hline \multicolumn{3}{|l|}{ Rotation $\left(^{\circ}\right)$} \\
\hline Type 1 & $0.028 \pm 0.543$ & $-0.049 \pm 0.491$ \\
\hline Type 2 & $-0.008 \pm 0.471$ & $-0.006 \pm 0.637$ \\
\hline Type 3 & $-0.075 \pm 0.482$ & $0.023 \pm 0.441$ \\
\hline$p$-value & 0.428 & 0.706 \\
\hline
\end{tabular}

Values are presented as mean \pm standard deviation. The one-way analysis of variance test is performed. Group A, placement of brackets with a customized resin base; Group B, placement of brackets without a customized resin base; Type 1, mild crowding; Type 2, moderate crowding; Type 3 , severe crowding.

The $p$-value is calculated using one-sample $t$-test; ${ }^{* *} p<0.01$, *** $p<0.001$. 
Table 3. Mutual comparison between mild, moderate, and severe crowding according to Little's irregularity index (group $A$ and $B$ )

\begin{tabular}{|c|c|c|c|}
\hline \multirow{3}{*}{ Variable } & \multicolumn{3}{|c|}{ Little's index (n) } \\
\hline & \multicolumn{2}{|c|}{ Type 1 (76) } & \multirow{2}{*}{$\begin{array}{l}\text { Type } 2 \text { (81) } \\
\text { Type } 3 \text { (68) }\end{array}$} \\
\hline & Type 2 (81) & Type 3 (68) & \\
\hline \multicolumn{4}{|l|}{ Group A } \\
\hline \multicolumn{4}{|c|}{ Mesio-distal (mm) } \\
\hline$\Delta \pm \mathrm{SD}$ & $-0.038 \pm 0.007$ & $-0.024 \pm 0.007$ & $0.011 \pm 0.007$ \\
\hline$p$-value & $<0.001^{* * *}$ & $0.002^{* *}$ & 0.389 \\
\hline \multicolumn{4}{|c|}{ Bucco-lingual (mm) } \\
\hline$\Delta \pm \mathrm{SD}$ & $0.002 \pm 0.008$ & $0.012 \pm 0.008$ & $0.010 \pm 0.008$ \\
\hline$p$-value & $>0.999$ & 0.438 & 0.758 \\
\hline \multicolumn{4}{|c|}{ Occluso-gingival (mm) } \\
\hline$\Delta \pm \mathrm{SD}$ & $-0.052 \pm 0.015$ & $-0.040 \pm 0.015$ & $0.009 \pm 0.015$ \\
\hline$p$-value & $0.002^{* *}$ & $0.021^{*}$ & $>0.999$ \\
\hline \multicolumn{4}{|l|}{ Torque $\left(^{\circ}\right)$} \\
\hline$\Delta \pm \mathrm{SD}$ & $0.022 \pm 0.107$ & $-0.001 \pm 0.111$ & $-0.025 \pm 0.109$ \\
\hline$p$-value & $>0.999$ & $>0.999$ & $>0.999$ \\
\hline \multicolumn{4}{|l|}{ Angulation $\left({ }^{\circ}\right)$} \\
\hline$\Delta \pm \mathrm{SD}$ & $-0.068 \pm 0.107$ & $-0.069 \pm 0.112$ & $0.009 \pm 0.110$ \\
\hline$p$-value & $>0.999$ & $>0.999$ & $>0.999$ \\
\hline \multicolumn{4}{|l|}{ Rotation $\left(^{\circ}\right)$} \\
\hline$\Delta \pm \mathrm{SD}$ & $0.036 \pm 0.080$ & $0.108 \pm 0.083$ & $0.066 \pm 0.082$ \\
\hline$p$-value & $>0.999$ & 0.728 & $>0.999$ \\
\hline \multicolumn{4}{|l|}{ Group B } \\
\hline \multicolumn{4}{|c|}{ Mesio-distal (mm) } \\
\hline$\Delta \pm \mathrm{SD}$ & $-0.006 \pm 0.005$ & $0.005 \pm 0.005$ & $0.012 \pm 0.005$ \\
\hline$p$-value & 0.728 & $>0.999$ & 0.093 \\
\hline \multicolumn{4}{|c|}{ Bucco-lingual (mm) } \\
\hline$\Delta \pm \mathrm{SD}$ & $0.037 \pm 0.009$ & $-0.004 \pm 0.009$ & $-0.039 \pm 0.009$ \\
\hline$p$-value & $0.001^{* *}$ & $>0.999$ & $<0.001^{* * *}$ \\
\hline \multicolumn{4}{|c|}{ Occluso-gingival (mm) } \\
\hline$\Delta \pm \mathrm{SD}$ & $-0.043 \pm 0.011$ & $-0.040 \pm 0.012$ & $-0.001 \pm 0.012$ \\
\hline$p$-value & $0.001^{* *}$ & $0.004^{* *}$ & $>0.999$ \\
\hline \multicolumn{4}{|l|}{ Torque $\left(^{\circ}\right)$} \\
\hline$\Delta \pm \mathrm{SD}$ & $0.161 \pm 0.104$ & $-0.037 \pm 0.109$ & $-0.184 \pm 0.107$ \\
\hline$p$-value & 0.373 & $>0.999$ & 0.266 \\
\hline \multicolumn{4}{|l|}{ Angulation $\left(^{\circ}\right)$} \\
\hline$\Delta \pm \mathrm{SD}$ & $0.030 \pm 0.122$ & $0.197 \pm 0.127$ & $0.168 \pm 0.126$ \\
\hline$p$-value & $>0.999$ & 0.370 & 0.545 \\
\hline \multicolumn{4}{|l|}{ Rotation $\left(^{\circ}\right)$} \\
\hline$\Delta \pm \mathrm{SD}$ & $-0.043 \pm 0.085$ & $-0.073 \pm 0.089$ & $-0.029 \pm 0.088$ \\
\hline$p$-value & $>0.999$ & $>0.999$ & $>0.999$ \\
\hline
\end{tabular}

Types 1, 2, and 3 represent mild, moderate, and severe crowding according to Little's irregularity index, respectively. $\mathrm{n}$ is the number of brackets used for analysis.

Group A, placement of brackets with a customized resin base; Group B, placement of brackets without a customized resin base; $\Delta$, mean difference; $\mathrm{SD}$, standard deviation.

The $p$-value is calculated using Bonferroni's post hoc test; ${ }^{*} p<0.05,{ }^{* *} p<0.01,{ }^{* * *} p<0.001$. 


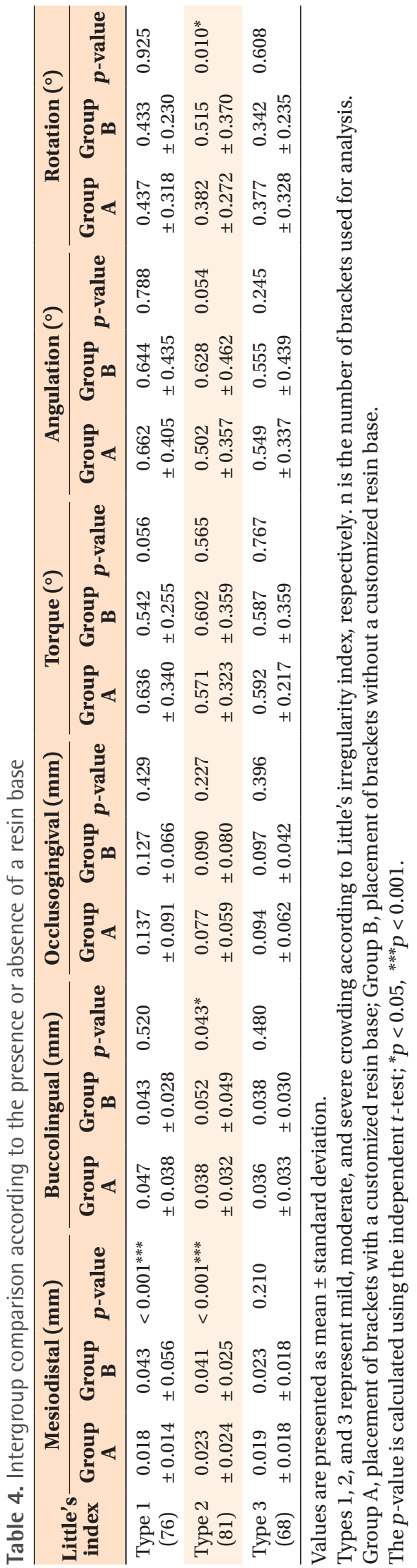

\section{DISCUSSION}

The positive or negative sign is determined according to the direction of the six measurement values for each bracket. If the descriptive statistics are calculated on this basis, the value could converge to zero under normally distributed characteristics of the sample. Although the results of the one-sample $t$-test for all the raw data are shown in Table 1, it is difficult to conclude that accuracy is guaranteed for all measured values without statistically significant differences. Instead, an absolute value for the raw data was obtained, and the value was considered the transfer discrepancy of the bracket position. In Figures 5 and 6, the measured absolute values were statistically significant in a 95\% confidence interval as revealed by the frequency and one-sided equivalence test for each deviation. The values were confirmed to be within a clinically acceptable accuracy range. For the linear measurements, compared to the origin, the average values showed a difference ranging from 0.029 to $0.101 \mathrm{~mm}$. For the angular measurements, rotation was the value that showed the least deviation, ranging from $0.396^{\circ}$ to $0.623^{\circ}$.

Directional bias is also an important factor in determining bracket transfer accuracy. Data on directional bias for each group are summarized in Figure 7. The biggest difference was that most of the brackets were biased toward the occlusal surface, regardless of the conditions in the $0-\mathrm{G}$ direction. In the case of torque, the bracket was attached in the direction where the crown was inclined towards the buccal surface. The reason for this vertical directional bias in the one-piece transfer jig could be an incorrectly positioned indirect transfer jig. Depending on whether the jig is completely seated on the incisal edge or occlusal surface of the tooth, a transfer discrepancy may or may not appear. When positioning the jig, the bonding agent is light-cured while applying a mild vertical finger pressure in the direction of the long axis of the tooth. ${ }^{27}$ The factors described above appear to have a mixed effect on the directional bias of torque. When finger pressure is applied from the buccal surface, the pressure may tip the bracket and the crown may tip to the lingual surface. If excess pressure and a thicker resin base are used, the jig itself may tilt to the lingual surface. Hence, the bracket is bonded in a direction that provides buccal crown torque. Lastly, the bracket has a tendency to attach towards the mesial surface in the $\mathrm{M}-\mathrm{D}$ direction.

In terms of crowding, the one-way ANOVA was performed for each group as shown in Tables 2 and 3. The analysis revealed no statistically significant difference in angular measurements regardless of the degree of crowding in both the groups or within the resin base groups. The dependent variables that were significantly 
A

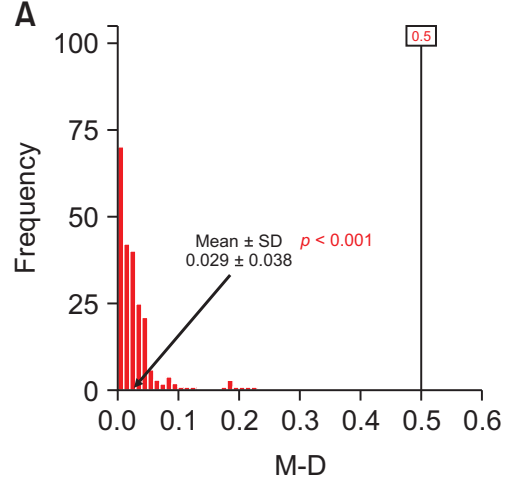

D

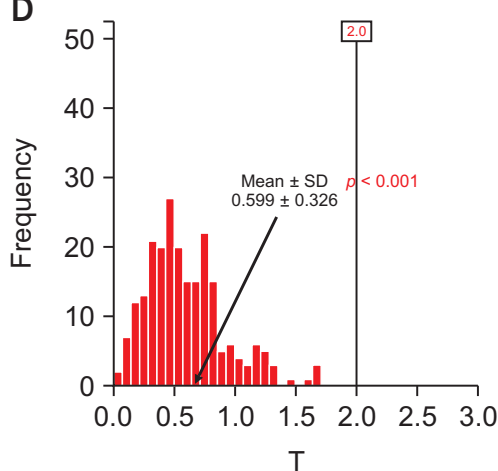

B

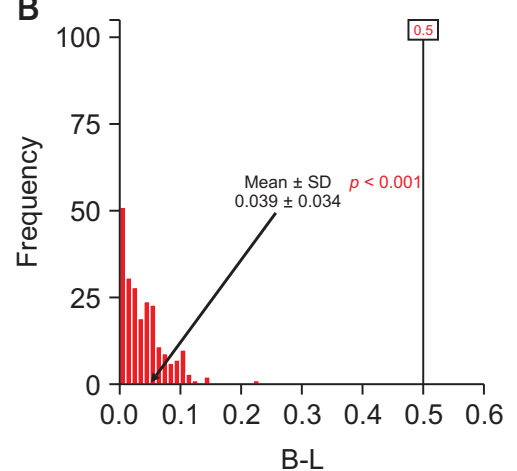

E

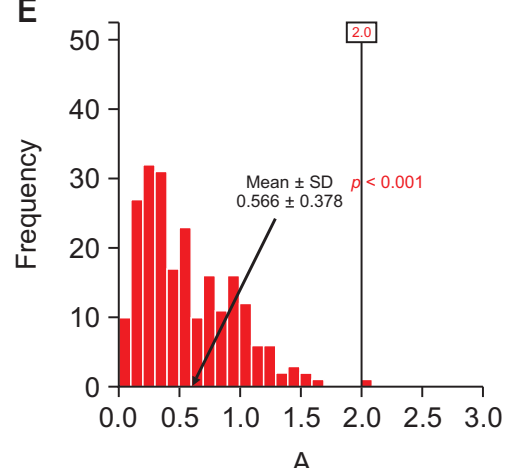

C

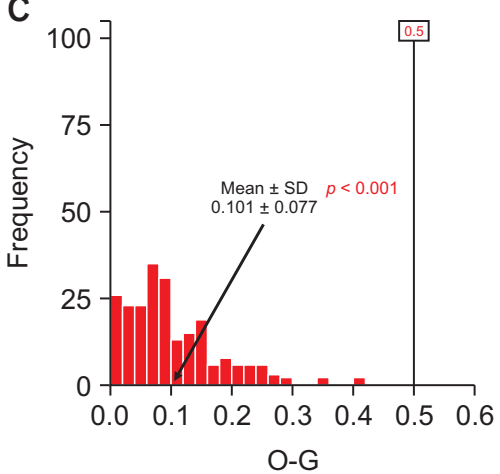

$\mathrm{F}$

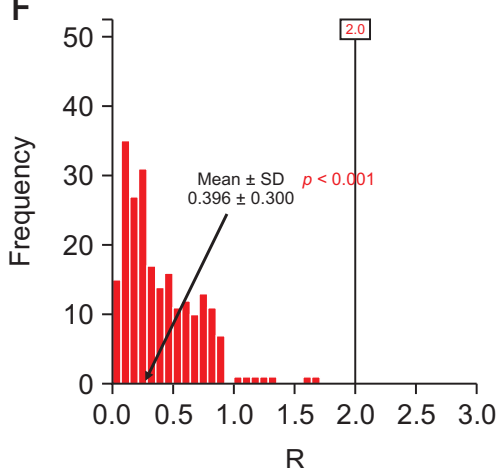

Figure 5. Histogram of frequencies for the six measurements generated using the one-tailed equivalence test for brackets with a customized resin base (group A). (A-C) linear and (D-F) angular measurements. Numbers on the horizontal axis indicate the differences between the virtual and actual models, and the height of each bar indicates the frequency of each difference range. The vertical line of the graph ( $0.5 \mathrm{~mm}$ in $\mathbf{A}-\mathbf{C}$, and $2.0^{\circ}$ in $\left.\mathbf{D}-\mathbf{F}\right)$ shows the American Board of Orthodontics Objective Grading System (ABO OGS) criterion. Almost all linear and angular measurements are within the $A B O$ OGS criterion. The $p$-value is calculated using the one-tailed equivalence test.

$M-D$, mesio-distal; $B-L$, bucco-lingual; $0-G$, occluso-gingival; $T$, torque; $A$, angulation; $R$, rotation; $S D$, standard deviation.

influenced according to Little's irregularity index were the M-D and $\mathrm{O}-\mathrm{G}$ in group $\mathrm{A}$. The post hoc test results revealed a significant difference as the crowding index increased from mild to moderate or from mild to severe (Table 3). In group B, significant differences occurred in the B-L and $\mathrm{O}-\mathrm{G}$ directions. These statistical differences were interpreted to be a result of Little's irregularity index becoming higher from mild to moderate crowding or from moderate to severe crowding in the B-L direction. However, a significant difference was observed between mild and moderate and/or mild and severe crowding in the 0-G direction after Bonferroni's post hoc test (Table 3).

Table 4 summarizes the effect of the resin base on each of the six dependent variables for the anterior teeth according to the degree of crowding. No significant difference was observed between the two groups in cases of severe crowding, because when the anterior teeth were severely crowded, some teeth could not attach to the bracket from the start of treatment. Paradoxically, a case of severe crowding is converted into a case without crowding. Therefore, a noticeable difference is observed when using the resin base in the values of M-D, $\mathrm{B}-\mathrm{L}$, and rotation in the moderate crowding group. In the mild crowding group, a significant difference was observed only in the M-D measurement. However, it is unreasonable to conclude that the dependent variables showing a deviation increase as the crowding increases, irrespective of the presence of the resin base. This suggests that the results are mixed regarding the interaction between the independent variables, such as the degree of crowding or presence of the resin base. However, when simply comparing the mean values, we concluded that the difference between the two groups according to the presence or absence of the resin base was not clinically meaningful.

The resin base allows the brackets to be customized to each individual tooth movement, but as the thickness increases, possible transfer errors may occur during laboratory processing or during the bracket bonding pro- 
A
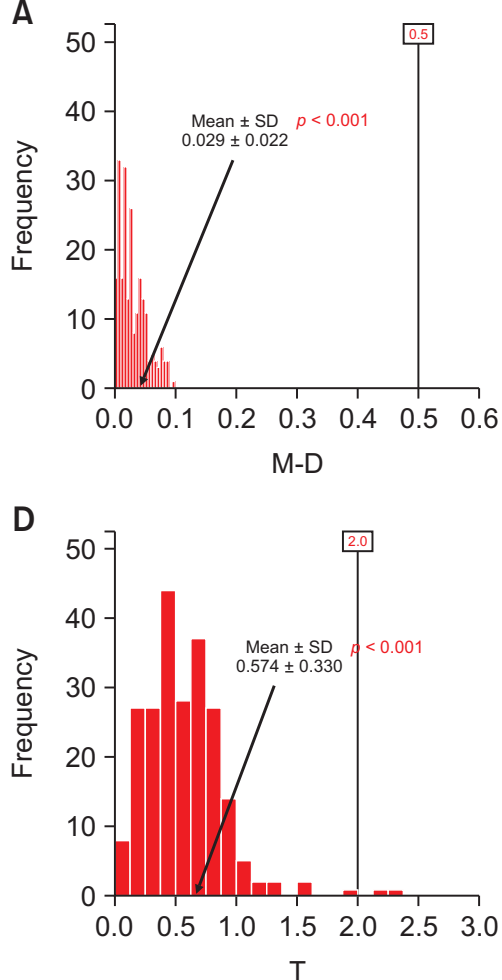

B

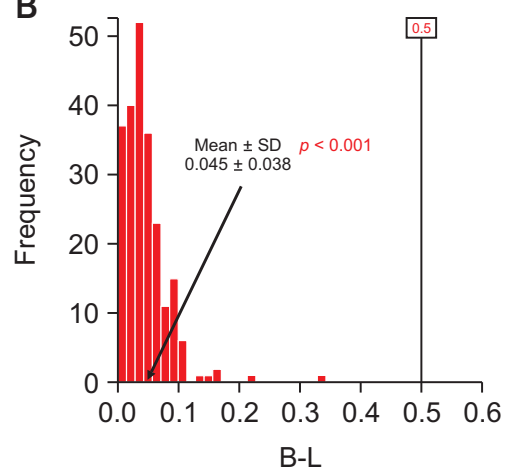

E

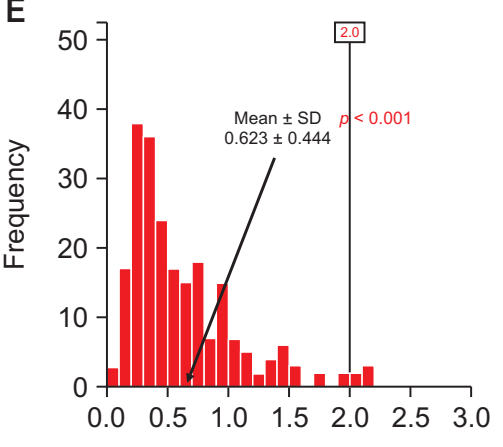

C

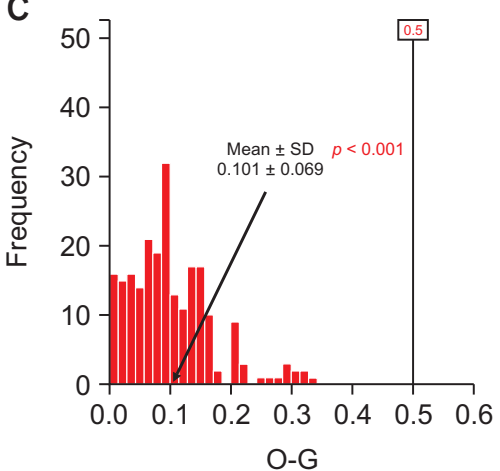

$\mathrm{F}$

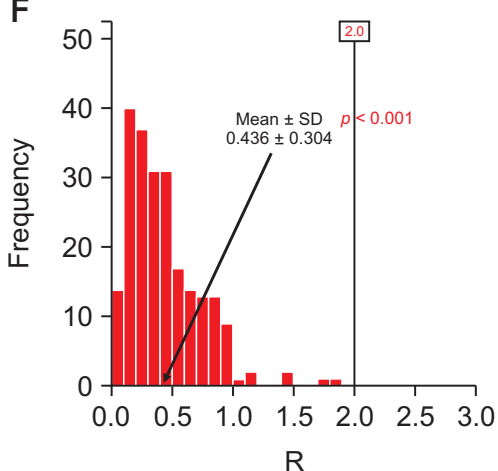

Figure 6. Histogram of frequencies for the six measurements generated using the one-tailed equivalence test for brackets without a customized resin base (group B). (A-C) linear and (D-F) angular measurements. Numbers on the horizontal axis indicate the differences between the virtual and actual models, and the height of each bar indicates the frequency of each difference range. The vertical line of the graph $\left(0.5 \mathrm{~mm}\right.$ in $\mathbf{A}-\mathbf{C}_{1}$ and $2.0^{\circ}$ in $\left.\mathbf{D}-\mathrm{F}\right)$ shows the American Board of Orthodontics Objective Grading System (ABO OGS) criterion. Almost all linear and angular measurements are within the $A B O$ OGS criterion. The $p$-value is calculated using the one-tailed equivalence test.

$M-D$, mesio-distal; $B-L$, bucco-lingual; $0-G$, occluso-gingival; $T$, torque; $A$, angulation; $R$, rotation; SD, standard deviation.
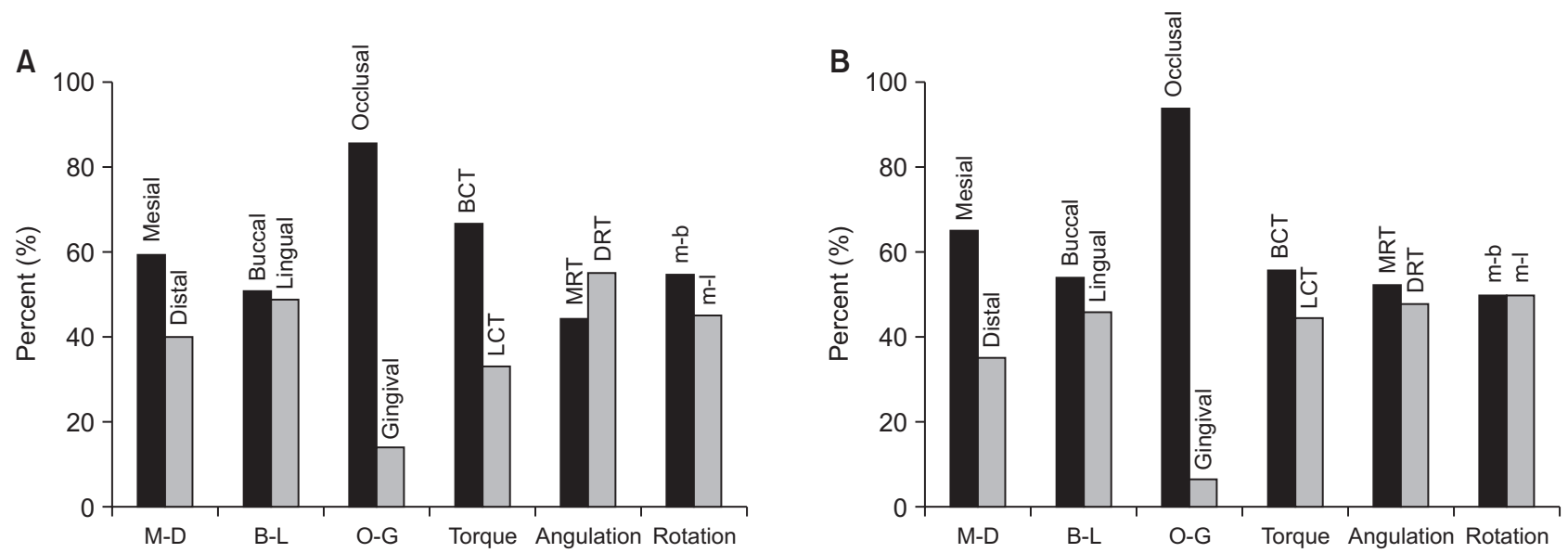

Figure 7. Percentages of frequencies of directional bias for the six measurements in group A (A) and group B (B).

$B C T$, buccal crown torque; LCT, lingual crown torque; MRT, mesial root tip; DRT, distal root tip; $m-b$, mesio-buccal; $m$-I, mesio-lingual; M-D, mesio-distal; B-L, bucco-lingual; O-G, occluso-gingival. 
cess. ${ }^{18,19}$ If the transfer device for IDBS has high accuracy without forming a resin base in advance, it is possible to eliminate the effort, time, and cost required in the laboratory, as well as to minimize errors. Since accuracy does not show any clinically meaningful difference according to the presence or absence of the resin base, the bracket transfer device in this study appears to have many advantages with respect to convenience.

Tooth shape and malformation could also affect transfer accuracy. A previous study suggested that the effect of cusp height was not clinically significant. ${ }^{20}$ We included only six anterior teeth in our study, and so the effect of the shape or cusp height of the posterior teeth was excluded. Nonetheless, we considered the differences in the shape and surface convexity of the canines to those of the central and lateral incisors. The result of this study showed that the accuracy of bracket position on the canines was not significantly different from that on the incisors. Thus, in our study, the shape of the canines could be ignored.

A one-piece jig was used in this study considering the accuracy of the material characteristics. The jig in this study was fabricated using plastic (VisiJet M3 Crystal; 3D Systems) with a tensile strength of $24.2 \mathrm{MPa}$, tensile modulus of 1,463 MPa, and flexural strength of $49 \mathrm{MPa}$. This higher level of material stiffness is inappropriate for a full arch transfer tray. A multiple bracket jig might be a faster alternative to using several one-piece jigs. However, the one-piece jig offers advantages such as the convenience of rebonding a single bracket in cases of bracket damage or failure. Comparing the accuracy of the one-piece jig to that of a transfer tray would be meaningful in a future study. In addition, comparing the results obtained using the current IDBS to other indirect bonding techniques would be interesting.

The application and accuracy of CAD/CAM in an IDBS have been studied. Poitter et al. ${ }^{28}$ compared the accuracy of a hard acrylic CAD/CAM tray to that of a soft onelayer silicone tray, and suggested that both trays were clinically acceptable but the silicone tray was more accurate than the hard acrylic CAD/CAM tray. Their hard acrylic CAD/CAM tray included multiple brackets, while we used the one-piece jig that accommodated only one bracket. This might have affected the accuracy.

A limitation of this study is that it is an in vitro experiment. In the clinical setting, more variables will have to be considered. Hence, to further confirm our results, an in vivo experiment would be valuable. Another limitation is the interaction between the variables, which could also affect the results.

\section{CONCLUSION}

Based on the findings of the study, we were able to conclude the following:

- The described indirect bonding jig could transfer anterior brackets exactly to the desired position of the patient's dentition within a clinically acceptable linear measurement range of $\pm 0.05 \mathrm{~mm}$ and an angular measurement range of $2.0^{\circ}$.

- Directional bias was most pronounced in the vertical direction, and many brackets were bonded slightly toward the occlusal surface. In addition, a clear tendency to transfer a bracket towards the buccal and mesial directions was observed. However, no apparent directional bias error was observed in the rotation direction.

- When considering the effect of crowding, statistical differences in the three angular measurement values (torque, angulation, and rotation) were not found in any group. However, differences in the vertical direction (0-G) were evident in both the groups.

- The effect of the resin base was not noticeable in the severe crowding group. In contrast, in the moderate crowding group, the measurement values $\mathrm{M}-\mathrm{D}, \mathrm{B}-\mathrm{L}$, and rotation were affected by the presence of the resin base.

\section{CONFLICTS OF INTEREST}

No potential conflict of interest relevant to this article was reported.

\section{ACKNOWLEDGEMENTS}

Yonsei University Industry-University Cooperation Foundation own a patent of one-body jig system (patent no. 10-2163625). We want to show our special thanks to Mr. Stefan Foerster General Manager of FORESTADENT Bernhard Förster GmbH, Pforzheim, Germany for supporting 0.022-inch Quicklear brackets for this research and Mr. Seung-Woo Kang, General manager of CENOS Co., Indeokwon, Gyeonggido, Korea for supporting manuscript preparation with virtual set-up, 3D printed Jig fabrication, and 3D-superimposition.

\section{REFERENCES}

1. Andrews LF. The straight-wire appliance. Explained and compared. J Clin Orthod 1976;10:174-95.

2. Andrews LF. The straight-wire appliance. Br J Orthod 1979;6:125-43.

3. Zachrisson BU, Brobakken BO. Clinical comparison of direct versus indirect bonding with different bracket types and adhesives. Am J Orthod 1978; 74:62-78.

4. Aguirre MJ, King GJ, Waldron JM. Assessment of bracket placement and bond strength when comparing direct bonding to indirect bonding techniques. Am J Orthod 1982;82:269-76.

5. Koo BC, Chung CH, Vanarsdall RL. Comparison of 
the accuracy of bracket placement between direct and indirect bonding techniques. Am J Orthod Dentofacial Orthop 1999;116:346-51.

6. Hodge TM, Dhopatkar AA, Rock WP, Spary DJ. A randomized clinical trial comparing the accuracy of direct versus indirect bracket placement. J Orthod 2004;31:132-7.

7. Thomas RG. Indirect bonding: simplicity in action. J Clin Orthod 1979;13:93-106.

8. Kalange JT. Indirect bonding: a comprehensive review of the advantages. World J Orthod 2004;5:3017.

9. Wendl B, Droschl H, Muchitsch P. Indirect bonding-a new transfer method. Eur J Orthod 2008;30:1007.

10. Lee MS. Indirect bonding of orthodontic brackets: an evaluation of transfer accuracy and reliability [Master's thesis]. Minnesota: University of Minnesota; 2014.

11. Castilla AE, Crowe JJ, Moses JR, Wang M, Ferracane JL, Covell DA Jr. Measurement and comparison of bracket transfer accuracy of five indirect bonding techniques. Angle Orthod 2014;84:607-14.

12. Seo H. Accuracy of indirect bracket bonding via virtual setup and 3D printing [Master's thesis]. Seoul: Yonsei University; 2016.

13. Niu Y, Zeng Y, Zhang Z, Xu W, Xiao L. Comparison of the transfer accuracy of two digital indirect bonding trays for labial bracket bonding. Angle Orthod 2021;91:67-73.

14. Gelin E, Seidel L, Bruwier A, Albert A, Charavet C. Innovative customized CAD/CAM nickel-titanium lingual retainer versus standard stainless-steel lingual retainer: a randomized controlled trial. Korean J Orthod 2020;50:373-82.

15. Koch PJ. Measuring the accuracy of a computeraided design and computer-aided manufacturingbased indirect bonding tray. Am J Orthod Dentofacial Orthop 2020;158:315.

16. Xue C, Xu H, Guo Y, Xu L, Dhami Y, Wang H, et al. Accurate bracket placement using a computer-aided design and computer-aided manufacturing-guided bonding device: an in vivo study. Am J Orthod Dentofacial Orthop 2020;157:269-77.

17. Kwon Sl, Lee KJ, inventor; CENOS assignee. Bracket jig system for positioning an orthodontic bracket.
Korea patent KR 10-2163625. 2020 Sep 29.

18. Fillion D. Clinical advantages of the Orapix-straight wire lingual technique. Int Orthod 2010;8:125-51.

19. Fillion D. Lingual straightwire treatment with the Orapix system. J Clin Orthod 2011;45:488-97; quiz 515.

20. Kim J, Chun YS, Kim M. Accuracy of bracket positions with a CAD/CAM indirect bonding system in posterior teeth with different cusp heights. Am J Orthod Dentofacial Orthop 2018;153:298-307.

21. Little RM. The irregularity index: a quantitative score of mandibular anterior alignment. Am J Orthod 1975;68:554-63.

22. Park KH, Choi JY, Kim KA, Kim SJ, Chung KR, Kim $\mathrm{SH}$. Critical issues concerning biocreative strategy in contemporary temporary skeletal anchorage device orthodontics: a narrative review. Orthod Craniofac Res 2021;24 Suppl 1:39-47.

23. Casko JS, Vaden JL, Kokich VG, Damone J, James $\mathrm{RD}$, Cangialosi TJ, et al. Objective grading system for dental casts and panoramic radiographs. American Board of Orthodontics. Am J Orthod Dentofacial Orthop 1998;114:589-99.

24. Wan Hassan WN, Yusoff Y, Mardi NA. Comparison of reconstructed rapid prototyping models produced by 3-dimensional printing and conventional stone models with different degrees of crowding. Am J Orthod Dentofacial Orthop 2017;151:209-18.

25. Hazeveld A, Huddleston Slater JJ, Ren Y. Accuracy and reproducibility of dental replica models reconstructed by different rapid prototyping techniques. Am J Orthod Dentofacial Orthop 2014;145:108-15.

26. Dietrich CA, Ender A, Baumgartner S, Mehl A. A validation study of reconstructed rapid prototyping models produced by two technologies. Angle Orthod 2017;87:782-7.

27. Gyllenhaal KA. Accuracy of two indirect bonding transfer methods: a three-dimensional, in-vivo analysis [Master's thesis]. Minnesota: University of Minnesota; 2015.

28. Pottier T, Brient A, Turpin YL, Chauvel B, Meuric V, Sorel 0 , et al. Accuracy evaluation of bracket repositioning by indirect bonding: hard acrylic CAD/CAM versus soft one-layer silicone trays, an in vitro study. Clin Oral Investig 2020;24:3889-97. 